\title{
ANALYTICAL FORM OF SHEPP-LOGAN PHANTOM FOR PARALLEL MRI
}

\author{
M. Guerquin-Kern ${ }^{1}$, F. I. Karahanoğlu ${ }^{2,3}$, D. Van De Ville $e^{2,3}$, K. P. Pruessmann ${ }^{4}$ and M. Unser ${ }^{1}$ \\ ${ }^{1}$ Biomedical Imaging Group, École Polytechnique Fédérale de Lausanne (EPFL), CH-1015 Lausanne \\ ${ }^{2}$ Medical Image Processing Lab, EPFL, CH-1015 Lausanne, CH-1211 Genève \\ ${ }^{3}$ Medical Image Processing Lab, Université de Genève, CH-1211 Genève \\ ${ }^{4}$ Institute for Biomedical Engineering, University and ETH Zurich, CH-8092 Zürich
}

\begin{abstract}
We present an analytical form of ground-truth $k$-space data for the 2-D Shepp-Logan brain phantom in the presence of multiple and non-homogeneous receiving coils. The analytical form allows us to conduct realistic simulations and validations of reconstruction algorithms for parallel MRI. The key contribution of our work is to use a polynomial representation of the coil's sensitivity. We show that this method is particularly accurate and fast with respect to the conventional methods. The implementation is made available to the community.
\end{abstract}

Index Terms - parallel magnetic resonance imaging, pMRI, phantom, Shepp-Logan, non-homogeneous coil, analytic simulation

\section{INTRODUCTION}

New reconstruction methods for magnetic resonance imaging (MRI) data have received a lot of attention in the field lately. Indeed, modern acquisition methodologies require to go beyond the traditional reconstruction scheme that is based on the inverse discrete Fourier transform (DFT). The current paradigm is to specify the reconstruction as an inverse problem [1]. One notable example is parallel MRI (pMRI), which exploits the presence of multiple receiver coils with different spatial sensitivity maps: SMASH [2, 3], SENSE [4], PILS [5], GRAPPA [6]. Another trend is the use of non-Cartesian $k$ space trajectories such as spiral $[7,8]$ or pseudo-random [9].

New MRI reconstruction algorithms obviously need to be evaluated and validated. Usually, the first stage - the quantitative evaluation - is based on experiments using synthetic data. The latter are generally derived from a numerically defined phantom, that provides reference data for reconstruction. The Shepp-Logan (SL) phantom is a widely-available candidate (see e.g. [10]) that was originally proposed for computed tomography. It is specified as a collection of ellipses with associated intensities. Mathematically, the phantom $\rho(\boldsymbol{r})$ is a linear combination of $R$ ellipses that encompass the regions $\mathcal{A}_{i}$ :

$$
\rho(\boldsymbol{r})=\sum_{i=1}^{R} \rho_{i} \mathbb{1}_{\mathcal{A}_{i}}(\boldsymbol{r}) .
$$

This work was supported in part by the Swiss National Competence Center in Biomedical Imaging (NCCBI), in part by the Center for Biomedical Imaging (CIBM), and in part by the Swiss National Science Foundation (grant PP00P2-123438).
Popular software tools (e.g. Matlab's Image Processing Toolkit) include an image-domain version of the SL phantom. For MRI, however, measurements are performed in $k$-space; i.e., the ideal measurement at position $\boldsymbol{k}$ corresponds to

$$
m(\boldsymbol{k})=\iint_{\mathbb{R}^{2}} \rho(\boldsymbol{r}) e^{\mathrm{j} 2 \pi k \cdot \boldsymbol{r}} \mathrm{d}^{2} \boldsymbol{r} .
$$

Synthesizing these data with straightforward spatial discretization combined with a DFT can generate significant aliasing artifacts. In fact, as the phantom is compactly supported in space, its energy density decreases relatively slowly for high frequencies.

An analytical form of the SL phantom in $k$-space has been proposed before. Smith et al. [11] raised the importance of accurate simulations for the quantitative validation of MR reconstructions. They proposed a protocol to generate the data but without entering in the details of the analytical formulation. Later, Van de Walle et al. [10] presented the analytical Fourier expression of the SL phantom as a combination of ellipses. Koay et al. [12] proposed a 3-D extension of the SL phantom adapted to MRI. Finally, Gach et al. [13] summarized and completed these works by proposing realistic parameters for the spin density and added the effect of $T_{1}$ and $T_{2}$ relaxation times to simulate even more realistic data. In all these works, the receiving coil was assumed to be homogeneous, making the simulations irrelevant to parallel MRI (pMRI). We are not aware of previously existing analytical phantoms suited to parallel MRI.

In parallel magnetic resonance imaging (pMRI), the model (2) that links the measurements to the phantom is adapted to take into account a complex-valued receiving coil sensitivity $S$ :

$$
m_{S}(\boldsymbol{k})=\iint_{\mathbb{R}^{2}} S(\boldsymbol{r}) \rho(\boldsymbol{r}) e^{\mathrm{j} 2 \pi \boldsymbol{k} \cdot \boldsymbol{r}} \mathrm{d}^{2} \boldsymbol{r} .
$$

The novelty in this work is to come up with an analytical expression for $m_{S}(\boldsymbol{k})$ for the case where the sensitivity function $S(\boldsymbol{r})$ is non-homogeneous. More precisely, we assume that it is represented by a complex-valued polynomial of degree $D$ inside the support of the phantom:

$$
S(\boldsymbol{r})=\sum_{d=0}^{D} \sum_{|\boldsymbol{\alpha}| \leq d} s_{d, \boldsymbol{\alpha}} \boldsymbol{r}^{\boldsymbol{\alpha}}, \quad \forall \boldsymbol{r} \in \bigcup_{i=1}^{R} \mathcal{A}_{i} .
$$

The analytical derivation of $m_{S}(\boldsymbol{k})$ is presented in Section 2 . 
In Section 3, we present some experiments to validate the method. In particular, we show that low polynomials are sufficient for the modeling of physically-realistic sensitivity profiles. We also check the consistency of our analytical computation with discretizations at increasing resolutions. It turns out that the analytical method is computationally competitive when a reasonable precision is required. Finally, we present an example of simulation of the acquisition of a Shepp-Logan phantom with different coils.

In this paper, we focus on the case of $2-\mathrm{D}$ pMRI as the proof-of-concept. However, there is no major theoretical obstacle that prevents extending the method to higher dimensions, including the temporal one (motion laws for the parameters of the phantoms, time-dependency of the k-space sampling and effect of $T_{1}$ and $T_{2}$ times).

For concise writing, we adopt the multi-index notations $z^{\alpha}=\prod z_{i}^{\alpha_{i}},|\boldsymbol{\alpha}|=\sum \alpha_{i}$ and $\sum_{p=\boldsymbol{a}}^{b}=\sum_{p_{1}=a_{1}}^{b_{1}} \sum_{p_{2}=a_{2}}^{b_{2}} \cdots$

\section{THEORY}

\subsection{Ellipses}

Let us consider the ellipse $\mathcal{A}$ centered on $\boldsymbol{r}_{c}$ with a rotation angle $\theta$. The change of coordinates $\boldsymbol{r} \mapsto \boldsymbol{u}$ that maps $\mathcal{A}$ into a unit disk is given by $\boldsymbol{r}=\boldsymbol{r}_{c}+\boldsymbol{R}(\theta) \boldsymbol{D} \boldsymbol{u}$ with $\boldsymbol{D}=\operatorname{diag}(A, B)$, where $A \geq B>0$ are the semi-major and semi-minor axes, and $\boldsymbol{R}(\theta)=\left[\begin{array}{cc}\cos (\theta) & -\sin (\theta) \\ \sin (\theta) & \cos (\theta)\end{array}\right]=\boldsymbol{R}^{-1}(-\theta)=\boldsymbol{R}^{\mathrm{T}}(-\theta)$, such that:

$$
\mathcal{A}=\{\boldsymbol{u} \mid\|\boldsymbol{u}\| \leq 1\}
$$

Figure 1 illustrates our conventions.
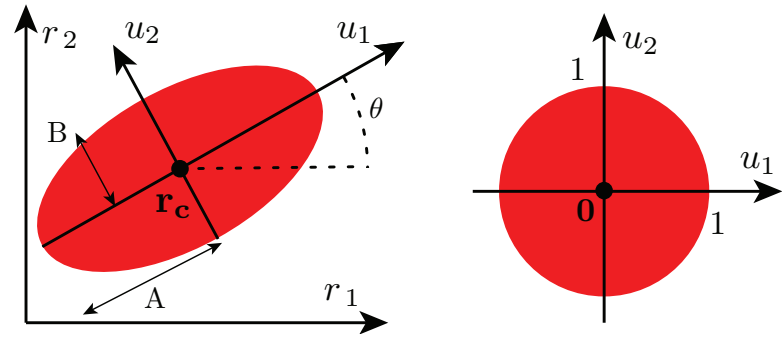

Fig. 1. Illustration of the change of coordinates to map an arbitrary ellipse into the unit disk.

\subsection{Inhomogeneous sensitivity}

For each ellipse, we decompose the polynomial coil sensitivity (cf. Eq. 4) in the new system of coordinates $\sum_{d=0}^{D} \sum_{|\boldsymbol{\alpha}| \leq d} t_{d, \boldsymbol{\alpha}} \boldsymbol{u}^{\boldsymbol{\alpha}}$, and rewrite the measurements as

$$
m_{S}(\boldsymbol{k})=\sum_{i=1}^{R} \rho_{i} \sum_{d=0}^{D} \sum_{|\boldsymbol{\alpha}| \leq d} t_{i, d, \boldsymbol{\alpha}} f_{\mathcal{A}}^{\boldsymbol{\alpha}}(-2 \pi \boldsymbol{k})
$$

with

$$
f_{\mathcal{A}}^{\boldsymbol{\alpha}}(\boldsymbol{\omega})=\iint_{\|\boldsymbol{u}\| \leq 1} \boldsymbol{u}^{\alpha} e^{-\mathrm{j} \boldsymbol{\omega} \cdot \boldsymbol{r}} \mathrm{d}^{2} \boldsymbol{r}
$$

We now propose a simple but practical way to implement the change of coordinate system.

In 2-D, there are $N_{p}=(D+1)(D+2) / 2$ complex-valued parameters $s_{d, \boldsymbol{\alpha}}$ and $t_{d, \boldsymbol{\alpha}}$, where $D$ is the polynomial degree. We build the $N_{s} \times N_{p}$ matrices $\boldsymbol{M}_{r}$ and $\boldsymbol{M}_{u}$ that relate the vectors of parameters $s$ and $\boldsymbol{t}$ to the value of the sensitivity at $N_{s} \geq N_{p}$ randomly chosen points $\boldsymbol{r}_{n}$, i.e. $\left[S\left(\boldsymbol{r}_{1}\right), \ldots, S\left(\boldsymbol{r}_{N}\right)\right]^{\mathrm{T}}=\boldsymbol{M}_{r} \boldsymbol{s}=\boldsymbol{M}_{u} \boldsymbol{t}$. The polynomial parameters $t_{d, \boldsymbol{\alpha}}$ are then determined as $\boldsymbol{t}=\boldsymbol{M}_{u}^{\dagger} \boldsymbol{M}_{r} \boldsymbol{s}^{1}$. Note that if the conditioning number of $\boldsymbol{M}_{u}$ is too high to ensure the stability of its inversion, the number $N_{s}$ of points can be increased.

\subsection{Partial differentials}

We define $G_{n}(\boldsymbol{x})=J_{n}(\|\boldsymbol{x}\|) /\|\boldsymbol{x}\|^{n}$ as a function of $\mathbb{R}^{2}$, where $J_{n}$ is the $n$-th order Bessel function of the first kind. $G_{n}$ is well-defined, continuous and infinitely differentiable on $\mathbb{R}^{2}$.

Proposition 1. For $\boldsymbol{\alpha} \in \mathbb{N}^{2}$ and $f_{\mathcal{A}}^{\boldsymbol{\alpha}}(\boldsymbol{\omega})$ defined by (7):

$$
f_{\mathcal{A}}^{\boldsymbol{\alpha}}(\boldsymbol{\omega})=2 \pi|\boldsymbol{D}| \mathrm{j}^{|\boldsymbol{\alpha}|} e^{-\mathrm{j} \boldsymbol{\omega} \cdot \boldsymbol{r}_{c}} \frac{\partial^{|\boldsymbol{\alpha}|} G_{1}}{\partial \boldsymbol{x}^{\alpha}}(\boldsymbol{D} \boldsymbol{R}(-\theta) \boldsymbol{\omega}) .
$$

Proof. First, we observe that $\iint_{\|\boldsymbol{u}\| \leq 1} e^{-\mathrm{j} \boldsymbol{x} \cdot \boldsymbol{u}} \mathrm{d}^{2} \boldsymbol{u}=2 \pi G_{1}(\boldsymbol{x})$ (see e.g. [10]). By differentiating with respect to $\boldsymbol{x}$, we get $\frac{\partial^{|\alpha|} G_{1}}{\partial \boldsymbol{x}^{\alpha}}(\boldsymbol{x})=\iint_{\|\boldsymbol{u}\| \leq 1}(-\mathrm{j} \boldsymbol{u})^{\alpha} e^{-\mathrm{j} \boldsymbol{x} \cdot \boldsymbol{u}} \mathrm{d}^{2} \boldsymbol{u}$. We obtain the result through a change of variable $\boldsymbol{r}=\boldsymbol{r}_{c}+\boldsymbol{R}(\theta) \boldsymbol{D} \boldsymbol{u}$, using the fact that $|\boldsymbol{R}(\theta) \boldsymbol{D}|=|\boldsymbol{D}|$.

The function $G_{n}$ has an interesting recursion property: $\nabla G_{n}(\boldsymbol{x})=-\boldsymbol{x} G_{n+1}(\boldsymbol{x})$. It follows that for $\boldsymbol{p} \in \mathbb{N}^{2}$ and $\boldsymbol{q} \in$ $\{0,1\}^{2}$, there exists a set of integer coefficients $C_{\boldsymbol{i}}^{\boldsymbol{p}, \boldsymbol{q}}, \boldsymbol{i}=$ 0...p p, such that:

$$
\frac{\partial^{|2 p+q|} G_{1}}{\partial \boldsymbol{x}^{2 \boldsymbol{p}+\boldsymbol{q}}}(\boldsymbol{x})=\boldsymbol{x}^{q} \sum_{i=0}^{p} C_{i}^{p, q} \boldsymbol{x}^{2 i} G_{|p+i+q|+1}(\boldsymbol{x}) .
$$

Then, it is fairly easy to write a program that recursively computes a lookup table of the coefficients $C_{i}^{p, q}$, starting from $C_{\mathbf{0}}^{\mathbf{0 , 0}}=1$.

\section{EXPERIMENTAL RESULTS}

In this Section, we present the experimental results. Our implementation was done using Matlab 7.9 (The MathWorks Inc. 2009, Natick) on a 64-bits 8-core computer, 4 GB RAM, Mac OS X 10.6. For comparison of execution times, it must be noted that most of the intensive computations, like the FFT, were distributed on the 8 cores. The code is made available online at http://bigwww.epfl.ch/algorithms. The Shepp-Logan brain phantom was considered as it is defined by default in Matlab, with a unitary dynamic range.

\subsection{Polynomial approximation of sensitivities}

In the first experiment, we tested the appropriateness of the polynomial representation for the sensitivity function. To that end, we simulated the (complex-valued) sensitivity in the 2-D imaging plane for several circular coils, according

\footnotetext{
${ }^{1}$ the superscript ${ }^{\dagger}$ denotes the Moore-Penrose pseudo-inverse
} 


\begin{tabular}{|c|c|c|c|c|c|c|c|c|c|c|}
\hline Degree & 1 & 2 & 3 & 4 & 5 & 6 & 7 & 8 & 9 & 10 \\
\hline \hline NRMSE & $1.34 \mathrm{e}-01$ & $9.63 \mathrm{e}-02$ & $6.48 \mathrm{e}-02$ & $4.10 \mathrm{e}-02$ & $2.44 \mathrm{e}-02$ & $1.38 \mathrm{e}-02$ & $8.02 \mathrm{e}-03$ & $5.31 \mathrm{e}-03$ & $4.00 \mathrm{e}-03$ & $3.23 \mathrm{e}-03$ \\
\hline SER $(\mathrm{dB})$ & 3.5 & 6.4 & 9.9 & 13.9 & 18.5 & 23.6 & 28.1 & $\mathbf{3 2 . 5}$ & $\mathbf{3 7 . 9}$ & $\mathbf{4 2 . 3}$ \\
\hline max. error & $7.2 \mathrm{e}-01$ & $5.1 \mathrm{e}-01$ & $3.3 \mathrm{e}-01$ & $2.1 \mathrm{e}-01$ & $1.4 \mathrm{e}-01$ & $8.7 \mathrm{e}-02$ & $6.1 \mathrm{e}-02$ & $4.7 \mathrm{e}-02$ & $3.8 \mathrm{e}-02$ & $3.1 \mathrm{e}-02$ \\
\hline
\end{tabular}

Table 1. Polynomial approximation of 12 head-coils sensitivities. Averaged values of normalized root mean square error, signal to error ratio and normalized maximum absolute error for different polynomial degrees.

to the Biot-Savart Law. We concentrated on the case when the coils are small and close to the sample, which is commonly the case in modern multichannel coils. Consequently, the sensitivity is highly varying in space. The parameters were chosen to reproduce the brain imaging conditions with receiving head-coils: field of view (FOV) of $28 \mathrm{~cm}, 12$ coils distributed around the skull, $15 \mathrm{~cm}$ distant from the center of the FOV and of radius $5 \mathrm{~cm}$. The pixelization for this simulation was $256 \times 256$. We fitted the polynomials in the least-squares fashion inside the support of the SL phantom (cf. Eq. 4) which included 27648 points. We report in Table 1 the fitting errors averaged over the 12 coils, for polynomial approximation up to degree 10.

As expected, the errors decreases drastically as the polynomial degree increases. We verified that these results are consistent for lower and larger pixelization resolutions. Given noise levels in a realistic multichannel setting and the difficulty of inversion (ill-posedness), $30 \mathrm{~dB}$ of SER is more that what can be generally expected for reconstruction. Therefore, we expect that polynomial degree greater than 8 should have low impact on the simulations and be sufficient for a faithful restitution of physical sensitivities.

\subsection{Discretization artifacts for pMRI simulation}

For the second experiment, we considered the error that results from the numerical generation of the data $m_{S}(\boldsymbol{k})$ by simple discrete Fourier transformation of a pixelized phantom. First, we chose a full Cartesian k-space trajectory supporting a $128 \times 128$ reconstruction matrix. With that trajectory, we looked particularly at the numerical errors in the reconstructed spatial domain. Second, we considered a nonCartesian spiral trajectory, always supporting a $128 \times 128$ reconstruction matrix. This time the goal was to figure out the impact of approximate non-Cartesian Fourier computations on the numerical measurements. For these computations, we used the NUFFT code provided by Fessler [14].

We considered the SL phantom with a single head-coil in the same configuration as described for the previous experiment. The polynomial sensitivity was of degree 8 (cf. 3.1).

We generated the numerical data with varying pixel resolutions and compared it with our analytical computations. The quality was evaluated in the space domain after applying the inverse DFT. The results are presented in Table 2 for the Cartesian sampling and in Table 3 for the spiral sampling.

As expected, the errors diminishes as the spatial resolution increases. Two cases could make computationally reasonable the choice of our analytical method: when few $k$-space samples are needed or when the trajectory supports a very large reconstruction image because the FFT routines of the numerical approach scale less well with increasing resolutions as it is observed in right part of Tables 2 and 3 (numbers in bold).

\begin{tabular}{|c|c|c|c|c|}
\hline \# pixels & $256^{2}$ & $512^{2}$ & $1024^{2}$ & $2048^{2}$ \\
\hline \hline NRMSE & $3.86 \mathrm{e}-03$ & $1.20 \mathrm{e}-03$ & $5.94 \mathrm{e}-04$ & $1.46 \mathrm{e}-04$ \\
\hline SER (dB) & 19.2 & 29.3 & 35.4 & 47.7 \\
\hline max. error & $9.27 \mathrm{e}-03$ & $3.21 \mathrm{e}-03$ & $1.27 \mathrm{e}-03$ & $3.45 \mathrm{e}-04$ \\
\hline max. err. space & $2.10 \mathrm{e}-01$ & $8.40 \mathrm{e}-02$ & $4.87 \mathrm{e}-02$ & $1.02 \mathrm{e}-02$ \\
\hline time (s) & 0.2 & 0.7 & 3.0 & 12.8 \\
\hline rel. time & 0.08 & 0.30 & $\mathbf{1 . 2 1}$ & $\mathbf{5 . 1 7}$ \\
\hline
\end{tabular}

Table 2. Numeric simulation with Cartesian sampling. For increasing resolutions: normalized root mean square error, signal to error ratio, relative maximal absolute error on the data and in the (reconstructed) image domain, time of computation and relative time execution w.r.t. the analytical method.

\begin{tabular}{|c|c|c|c|c|}
\hline \# pixels & $256^{2}$ & $512^{2}$ & $1024^{2}$ & $2048^{2}$ \\
\hline \hline NRMSE & $3.49 \mathrm{e}-03$ & $1.08 \mathrm{e}-03$ & $5.36 \mathrm{e}-04$ & $1.32 \mathrm{e}-04$ \\
\hline SER (dB) & 19.0 & 29.1 & 35.2 & 47.4 \\
\hline max. error & $8.52 \mathrm{e}-03$ & $2.79 \mathrm{e}-03$ & $1.16 \mathrm{e}-03$ & $3.07 \mathrm{e}-04$ \\
\hline time (s) & 2.3 & 2.2 & 4.8 & 14.9 \\
\hline rel. time & 0.93 & 0.92 & $\mathbf{1 . 9 6}$ & $\mathbf{6 . 1 4}$ \\
\hline
\end{tabular}

Table 3. Numeric simulation with spiral sampling. For increasing resolutions: normalized root mean square error, signal to error ratio, relative maximal absolute error of the simulation, time of computation and relative time execution w.r.t. the analytical method.

Finally, for illustration purposes, we simulated a 3channel pMRI scan of the SL phantom with full Cartesian sampling supporting a reconstruction of size $256 \times 256$. The analytical computations required $35 \mathrm{~s}$ for a full Cartesian sampling supporting $256 \times 256$ resolution and a polynomial degree 8 . The analytical data transformed in space domain (after inverse DFT) are shown together with the difference to the direct pixelization of the phantom. Space domain data and differences (modulus of) are presented in Fig. 2. They exhibit ringing artifacts due to insufficient high-frequency sampling.

\section{CONCLUSION}

We presented an extended analytical model of the famous Shepp-Logan phantom that is suitable for pMRI simulation. The model is able to incorporate the sensitivity maps of the receiving coils. The analytical computations allowed to measure the error that is done when using numerical simulations. It turns out that this error is not negligible when using small resolutions. On the contrary, these numerical computation seem accurate enough for high resolutions. How- 

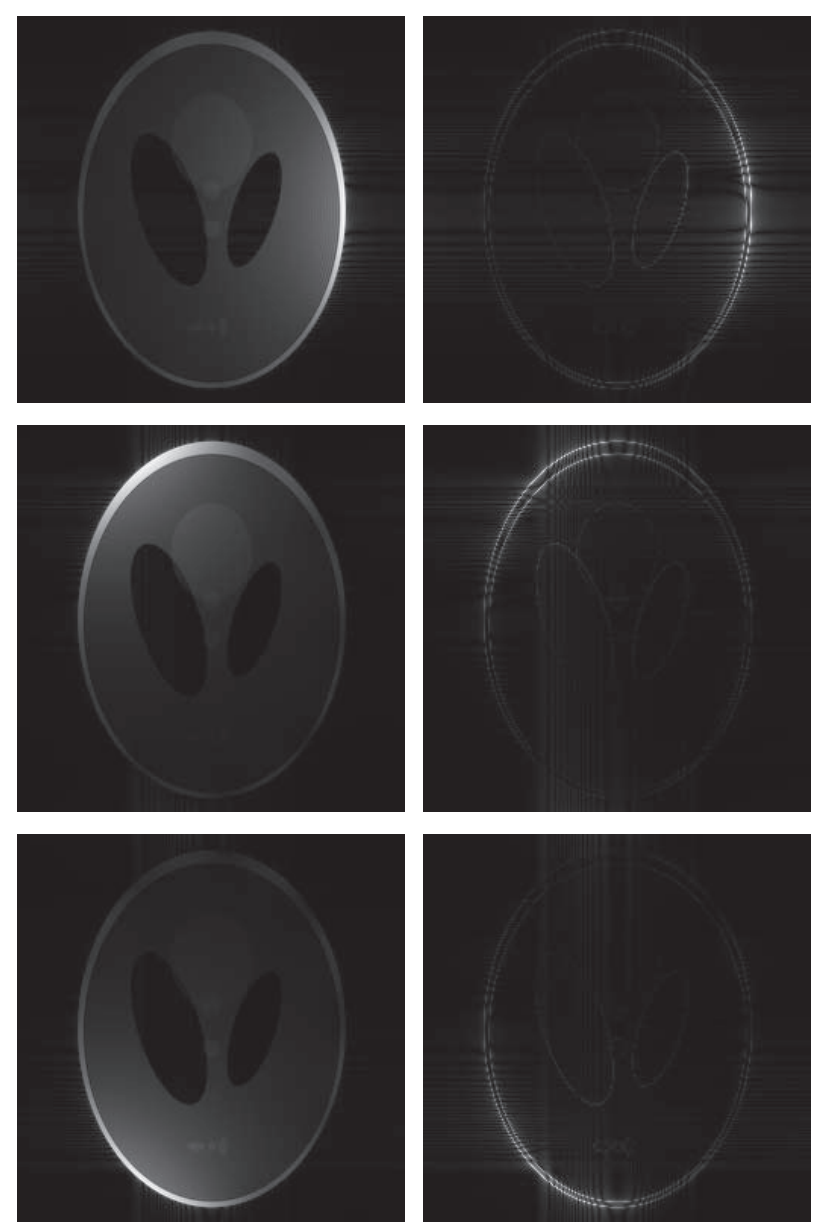

Fig. 2. Reconstructed images after inverse Fourier transform of simulated full k-space data for 3 coil profiles (left column) and differences with the direct discretization (right column).

ever, the available memory limits the resolution and the execution time increases significantly. That is why our analytical approach is a good alternative to numerical simulations. The Matlab code is made available on the website http://bigwww.epfl.ch/algorithms, such that all the experiments are reproducible and this work could benefit people validating and comparing pMRI reconstruction software.

As further extensions to this work, let us mention directions towards a more accurate model: taking into account relaxation times [13], diversification of the phantom to higher dimensions including time or towards more shapes available for analytical computations [15].

\section{REFERENCES}

[1] A. Ribés and F. Schmitt, "Linear inverse problems in imaging," IEEE Signal Processing Magazine, vol. 25, no. 4, pp. 84-99, July 2008.

[2] D. K. Sodickson and W. J. Manning, "Simultaneous acquisition of spatial harmonics (SMASH): fast imaging with radiofrequency coil arrays," Magnetic Resonance in Medicine, vol. 38, no. 4, pp. 591-603, 1997.

[3] P. M. Jakob, M. A. Griswold, R. R. Edelman, and D. K. Sodickson, "AUTO-SMASH: a self-calibrating technique for SMASH imaging," Magnetic Resonance Materials in Physics, Biology and Medicine, vol. 7, no. 1, pp. 42-54, 1998.

[4] K. P. Pruessmann, M. Weiger, M. B. Scheidegger, and P. Boesiger, "SENSE: Sensitivity encoding for fast MRI," Magnetic Resonance in Medicine, vol. 42, no. 5, pp. 952-962, 1999.

[5] M. A. Griswold, P. M. Jakob, M. Nittka, J. W. Goldfarb, and A. Haase, "Partially parallel Imaging with Localized Sensitivities (PILS)," Magnetic Resonance in Medicine, vol. 44, no. 4, pp. 602-609, 2000.

[6] M. A. Griswold, P. M. Jackob, R. M. Heidemann, M. Nittka, V. Jellus, J. Wang, B. Kiefer, and A. Haase, "GeneRalized Autocalibrating Partially Parallel Acquisition (GRAPPA)," Magnetic Resonance in Medicine, vol. 47, no. 6, pp. 1202-1210, 2002.

[7] G. H. Glover, "Simple analytic spiral k-space algorithm," Magnetic Resonance in Medicine, vol. 42, no. 2, pp. 412415, 1999.

[8] D.-H. Kim, E. Adalsteinsson, and D. M. Spielman, "Simple analytic variable density spiral design," Magnetic Resonance in Medicine, vol. 50, no. 1, pp. 214-219, 2003.

[9] M. Lustig, D. L. Donoho, and J. M. Pauly, "Sparse MRI: The application of compressed sensing for rapid MR imaging," Magnetic Resonance in Medicine, vol. 58, pp. 1182-1195, 2007.

[10] R. Van de Walle, H. H. Barrett, K. J. Myers, M. I. Aitbach, B. Desplanques, A. F. Gmitro, J. Cornelis, and I. Lemahieu, "Reconstruction of MR images from data acquired on a general nonregular grid by pseudoinverse calculation," vol. 19, no. 12, pp. 1160-1167, 2000.

[11] M. R. Smith, L. Chen, Y. Hui, T. Mathews, J. Yang, and X. Zeng, "Alternatives to the use of the DFT in MRI and spectroscopic reconstructions," International Journal of Imaging Systems and Technology, vol. 8, no. 6, pp. 558-564, 1997.

[12] C. G. Koay, J. E. Sarlls, and E. Ãzarslan, "Threedimensional analytical magnetic resonance imaging phantom in the Fourier domain," Magnetic Resonance in Medicine, vol. 58, no. 2, pp. 430-436, August 2007.

[13] M. H. Gach, C. Tanase, and F. Boada, "2D \& 3D SheppLogan phantom standards for MRI," in International Conference on Systems Engineering, Los Alamitos, CA, USA, 2008, pp. 521-526, IEEE Computer Society.

[14] J. A. Fessler and B. P. Sutton, "Nonuniform fast Fourier transforms using min-max interpolation," IEEE Transactions on Signal Processing, vol. 51, no. 2, pp. 560-574, 2003.

[15] F. I. Karahanoglu, "Design of a new hearth phantom for cardiac MRI simulations," M.S. thesis, École Polytechnique Fédérale de Lausanne, 2008. 